\title{
On an equation characterizing multi-Jensen-quadratic mappings and its Hyers-Ulam stability via a fixed point method
}

\author{
Anna Bahyrycz and Krzysztof Ciepliński
}

\begin{abstract}
In this paper, we unify the system of functional equations defining a multi-Jensen-quadratic mapping to obtain a single equation. We also prove, using the fixed point method, the generalized HyersUlam stability of this equation both in Banach spaces and in complete non-Archimedean normed spaces.
\end{abstract}

Mathematics Subject Classification. 39B52, 39B72, 39B82, 47H10.

Keywords. Multi-Jensen-quadratic mapping, (generalized) Hyers-Ulam stability, fixed point method, non-Archimedean space.

\section{Introduction}

It is well known that among functional equations, the Jensen equation

$$
f\left(\frac{x+y}{2}\right)=\frac{f(x)+f(y)}{2}
$$

(which is closely connected with the notion of convex function) and the Jordan-von Neumann (quadratic) equation

$$
q(x+y)+q(x-y)=2 q(x)+2 q(y)
$$

(which is useful in some characterizations of inner product spaces) play a prominent role. A lot of information about their solutions (which are said to be Jensen and quadratic mappings, respectively), stability and applications can be found for instance in [21, 24, 28].

Let us recall that a group $G$ is called uniquely divisible by 2 provided that for every $x \in G$ there exists a unique $y \in G$ (which is denoted by $\frac{x}{2}$ or $\left.\frac{1}{2} x\right)$ such that $x=2 y$. Given two groups $G$ and $H$ which are uniquely divisible 
by 2, we say (see also [9]) that a function $f: G^{n} \rightarrow H$ is $k$-Jensen and $n-k$ quadratic (briefly, multi-Jensen-quadratic) if $f$ satisfies Jensen's functional equation in each of some $k$ variables and the Jordan-von Neumann equation in each of the other variables. In this paper, we suppose for simplicity that $f$ fulfils Jensen's equation in each of the first $k$ variables, but one can obtain analogous results without this assumption.

Let us note that for $k=n$ the above definition leads to the so-called multi-Jensen mappings (introduced in 2005 by Prager and Schwaiger [26] with the connection with generalized polynomials); for $k=0$ we obtain the notion of multi-quadratic function (see [14]); a 1-Jensen and 1-quadratic mapping is just a Jensen-quadratic mapping defined by Bae and Park in [1].

In this paper, we reduce the system of $n$ equations defining the multiJensen-quadratic mapping to a single functional equation and we prove the generalized (in the spirit of D. G. Bourgin and P. Găvruţa) Hyers-Ulam stability of this equation both in Banach spaces and in complete non-Archimedean normed spaces.

Let us recall that speaking of the stability of a functional equation we follow the question raised in 1940 by Ulam and the first partial answer (in the case of Cauchy's equation in Banach spaces) to it given by Hyers. After Hyers' result a great number of papers (see for instance $[2,5,6,10,11,15$, $17,19,20,21]$ and the references therein) on the subject has been published, generalizing Ulam's problem and Hyers' theorem in various directions and to other (not only functional) equations.

The first work on the Hyers-Ulam stability of functional equations in complete non-Archimedean normed spaces (some particular cases were considered earlier; see [5] for details) is [25]. After it a lot of papers (see for instance $[8,16,30]$ and the references therein) on the stability of other equations in such spaces have been published.

In the proofs of our stability results (Theorems 3.3 and 3.4) we use the fixed point method, which was used for the investigation of the Hyers-Ulam stability of functional equations for the first time by J. A. Baker. For more information about this method we refer the reader to $[2,5,6,15,17]$ and the references therein.

\section{Preliminaries}

Throughout this paper, $\mathbb{N}$ stands for the set of all positive integers, $\mathbb{N}_{0}:=$ $\mathbb{N} \cup\{0\}, \mathbb{R}_{+}:=[0, \infty), n \in \mathbb{N}$ and $k \in\{0, \ldots, n\}$. Moreover, given a nonempty set $V$, we identify $x=\left(x_{1}, \ldots, x_{n}\right) \in V^{n}$ with $\left(x^{1}, x^{2}\right) \in V^{k} \times V^{n-k}$, where $x^{1}:=\left(x_{1}, \ldots, x_{k}\right)$ and $x^{2}:=\left(x_{k+1}, \ldots, x_{n}\right)$.

For any $l \in \mathbb{N}_{0}, m \in \mathbb{N}, t=\left(t_{1}, \ldots, t_{m}\right) \in\{-2,-1,0,1,2\}^{m}$ and $x=$ $\left(x_{1}, \ldots, x_{m}\right) \in V^{m}$, we write

$$
l x:=\left(l x_{1}, \ldots, l x_{m}\right) \text { and } t x:=\left(t_{1} x_{1}, \ldots, t_{m} x_{m}\right),
$$

where $r a$ stands, as usual, for the $r$ th power of an element $a$ of the commutative group $V$. Finally, we adopt the convention that for any set $A, A^{0}:=\emptyset$. 
Now, we recall some definitions and facts which will be needed in what follows.

We start with a fixed point result that can be derived from [7, Theorem 1]. In order to do this, we introduce the following three hypotheses.

(H1) $E$ is a nonempty set, $Y$ is a Banach space, $j \in \mathbb{N}, f_{1}, \ldots, f_{j}: E \rightarrow E$ and $L_{1}, \ldots, L_{j}: E \rightarrow \mathbb{R}_{+}$.

(H2) $\mathcal{T}: Y^{E} \rightarrow Y^{E}$ is an operator satisfying the inequality

$$
\begin{aligned}
\| \mathcal{T} \xi(x) & -\mathcal{T} \mu(x) \| \\
& \leq \sum_{i=1}^{j} L_{i}(x)\left\|\xi\left(f_{i}(x)\right)-\mu\left(f_{i}(x)\right)\right\|, \quad \xi, \mu \in Y^{E}, x \in E .
\end{aligned}
$$

(H3) $\Lambda: \mathbb{R}_{+}^{E} \rightarrow \mathbb{R}_{+}^{E}$ is an operator defined by

$$
\Lambda \delta(x):=\sum_{i=1}^{j} L_{i}(x) \delta\left(f_{i}(x)\right), \quad \delta \in \mathbb{R}_{+}^{E}, x \in E .
$$
theorem.

Now, we are in a position to present the above-mentioned fixed point

Theorem 2.1. Let hypotheses (H1)-(H3) hold and the functions $\varepsilon: E \rightarrow \mathbb{R}_{+}$ and $\varphi: E \rightarrow Y$ fulfill the following two conditions:

$$
\begin{gathered}
\|\mathcal{T} \varphi(x)-\varphi(x)\| \leq \varepsilon(x), \quad x \in E, \\
\varepsilon^{*}(x):=\sum_{l=0}^{\infty} \Lambda^{l} \varepsilon(x)<\infty, \quad x \in E .
\end{gathered}
$$

Then there exists a unique fixed point $\psi$ of $\mathcal{T}$ with

$$
\|\varphi(x)-\psi(x)\| \leq \varepsilon^{*}(x), \quad x \in E .
$$

Moreover,

$$
\psi(x)=\lim _{l \rightarrow \infty} \mathcal{T}^{l} \varphi(x), \quad x \in E .
$$

Given an $m \in \mathbb{N}$ we write $S:=\{0,1\}^{m}$, and $S_{i}$ stands for the set of all elements of $S$ having exactly $i$ zeros; i.e.,

$$
S_{i}:=\left\{\left(s_{1}, \ldots, s_{m}\right) \in S: \operatorname{card}\left\{j: s_{j}=0\right\}=i\right\}, \quad i \in\{0, \ldots, m\} .
$$

Moreover, for any $l \in \mathbb{N}_{0}, s=\left(s_{1}, \ldots, s_{m}\right), t=\left(t_{1}, \ldots, t_{m}\right) \in\{-2,-1,0,1,2\}^{m}$ we put

$$
l t:=\left(l t_{1}, \ldots, l t_{m}\right) \text { and } s t:=\left(s_{1} t_{1}, \ldots, s_{m} t_{m}\right) .
$$

The following technical lemma from [3] will also be useful in the proof of our first stability result.

Lemma 2.2. If $m \in \mathbb{N}, l \in \mathbb{N}_{0}$ and $\varphi: S \rightarrow \mathbb{R}_{+}$, then

$$
\sum_{v=0}^{m} \sum_{w=0}^{m} \sum_{s \in S_{w}} \sum_{t \in S_{v}}\left(2^{l}-1\right)^{w} \varphi(s t)=\sum_{i=0}^{m} \sum_{p \in S_{i}}\left(2^{l+1}-1\right)^{i} \varphi(p) .
$$


Let us next recall (see for instance [22]) some basic definitions and facts concerning non-Archimedean normed spaces.

By a non-Archimedean field we mean a field $\mathbb{K}$ equipped with a function (called valuation) $|\cdot|: \mathbb{K} \rightarrow \mathbb{R}_{+}$such that

$$
\begin{aligned}
|r| & =0 \quad \text { if and only if } \quad r=0, \\
|r s| & =|r||s|, \quad r, s \in \mathbb{K}
\end{aligned}
$$

and

$$
|r+s| \leq \max \{|r|,|s|\}, \quad r, s \in \mathbb{K}
$$

In any non-Archimedean field we have $|1|=|-1|=1$ and $|n| \leq 1$ for $n \in \mathbb{N}_{0}$.

In any field $\mathbb{K}$ the function $|\cdot|: \mathbb{K} \rightarrow \mathbb{R}_{+}$given by

$$
|x|:= \begin{cases}0, & x=0 \\ 1, & x \neq 0,\end{cases}
$$

is a valuation which is called trivial, but the most important examples of non-Archimedean fields are $p$-adic numbers which have gained the interest of physicists for their research in some problems coming from quantum physics, $p$-adic strings and superstrings.

Let $X$ be a linear space over a field $\mathbb{K}$ with a non-Archimedean nontrivial valuation $|\cdot|$. A function $\|\cdot\|: X \rightarrow \mathbb{R}_{+}$is said to be a non-Archimedean norm if it satisfies the following conditions:

$$
\begin{aligned}
\|x\| & =0 \quad \text { if and only if } \quad x=0, \\
\|r x\| & =|r|\|x\|, \quad r \in \mathbb{K}, x \in X,
\end{aligned}
$$

and

$$
\|x+y\| \leq \max \{\|x\|,\|y\|\}, \quad x, y \in X .
$$

Then $(X,\|\cdot\|)$ is called a non-Archimedean normed space.

In any such a space the function $d: X \times X \rightarrow \mathbb{R}_{+}$given by

$$
d(x, y)=\|x-y\|, \quad x, y \in X
$$

is a metric on $X$. Recall also that a sequence $\left(x_{n}\right)_{n \in \mathbb{N}}$ of elements of a nonArchimedean normed space is Cauchy if and only if $\left(x_{n+1}-x_{n}\right)_{n \in \mathbb{N}}$ converges to zero. Moreover, the addition, scalar multiplication and non-Archimedean norm are continuous mappings.

Finally, we give another fixed point result that can be derived from $[8$, Theorem 1]. In order to do this, we introduce the following three hypotheses.

(N1) $E$ is a nonempty set, $Y$ is a complete non-Archimedean normed space over a non-Archimedean field of a characteristic different from $2, j \in$ $\mathbb{N}, f_{1}, \ldots, f_{j}: E \rightarrow E$ and $L_{1}, \ldots, L_{j}: E \rightarrow \mathbb{R}_{+}$. 
(N2) $\mathcal{T}: Y^{E} \rightarrow Y^{E}$ is an operator satisfying the inequality

$$
\begin{aligned}
\| \mathcal{T} \xi(x) & -\mathcal{T} \mu(x) \| \\
& \leq \max _{i \in\{1, \ldots, j\}} L_{i}(x)\left\|\xi\left(f_{i}(x)\right)-\mu\left(f_{i}(x)\right)\right\|, \quad \xi, \mu \in Y^{E}, x \in E .
\end{aligned}
$$

(N3) $\Lambda: \mathbb{R}_{+}^{E} \rightarrow \mathbb{R}_{+}^{E}$ is an operator defined by

$$
\Lambda \delta(x):=\max _{i \in\{1, \ldots, j\}} L_{i}(x) \delta\left(f_{i}(x)\right), \quad \delta \in \mathbb{R}_{+}^{E}, x \in E .
$$

Now, we are in a position to present the mentioned fixed point theorem.

Theorem 2.3. Let hypotheses (N1)-(N3) hold and the functions $\varepsilon: E \rightarrow \mathbb{R}_{+}$ and $\varphi: E \rightarrow Y$ fulfill condition (2.1) and

$$
\lim _{l \rightarrow \infty} \Lambda^{l} \varepsilon(x)=0, \quad x \in E .
$$

Then for every $x \in E$ the limit

$$
\lim _{l \rightarrow \infty} \mathcal{T}^{l} \varphi(x)=: \psi(x)
$$

exists and the function $\psi \in Y^{E}$, defined in this way, is a fixed point of $\mathcal{T}$ with

$$
\|\varphi(x)-\psi(x)\| \leq \sup _{l \in \mathbb{N}_{0}} \Lambda^{l} \varepsilon(x), \quad x \in E .
$$

\section{Results}

\subsection{A characterization of multi-Jensen-quadratic mappings}

First, we reduce the system of $n$ equations defining the $k$-Jensen and $n-k$ quadratic mapping to obtain a single functional equation. In order to do this, we will use the following lemma.

Lemma 3.1. Let $V$ be a commutative group uniquely divisible by $2, W$ a linear space over the rationals, $n \in \mathbb{N}$ and $k \in\{0, \ldots, n-1\}$. If $f: V^{n} \rightarrow W$ satisfies, for any $x_{i}^{1}:=\left(x_{1 i}, \ldots, x_{k i}\right) \in V^{k}, x_{i}^{2}:=\left(x_{k+1 i}, \ldots, x_{n i}\right) \in V^{n-k}$, $i \in\{1,2\}$, the equation

$$
\sum_{q \in\{-1,1\}^{n-k}} f\left(\frac{x_{1}^{1}+x_{2}^{1}}{2}, x_{1}^{2}+q x_{2}^{2}\right)=2^{n-2 k} \sum_{i_{1}, \ldots, i_{n} \in\{1,2\}} f\left(x_{1 i_{1}}, \ldots, x_{n i_{n}}\right),
$$

then $f(x)=0$ for any $x=\left(x^{1}, x^{2}\right) \in V^{n}$ such that at least one component of $x^{2}$ is equal to zero.

Proof. Putting $x_{1}^{1}=x_{2}^{1}=x^{1}$ and $x_{1}^{2}=x_{2}^{2}=(0, \ldots, 0)$ in (3.1) we get

$$
2^{n-k} f\left(x^{1}, 0, \ldots, 0\right)=2^{n-2 k} 2^{n} f\left(x^{1}, 0, \ldots, 0\right),
$$

and consequently $f\left(x^{1}, 0, \ldots, 0\right)=0$.

If $n-k \geq 2$, then we fix $j \in\{k+1, \ldots, n\}, x_{j 1} \in V$ and put $x_{j 2}=$ $x_{m i_{m}}=0$, where $i_{m} \in\{1,2\}$, for $m \in\{k+1, \ldots, n\} \backslash\{j\}$ and $x_{1}^{1}=x_{2}^{1}=x^{1}$. Then, by (3.1),

$$
2^{n-k} f\left(x^{1}, 0, \ldots, 0, x_{j 1}, 0, \ldots, 0\right)=2^{n-2 k} 2^{n-1} f\left(x^{1}, 0, \ldots, 0, x_{j 1}, 0, \ldots, 0\right),
$$


and thus

$$
f\left(x^{1}, 0, \ldots, 0, x_{j 1}, 0, \ldots, 0\right)=0 .
$$

We continue in this fashion obtaining $f(x)=0$ for any $x=\left(x^{1}, x^{2}\right) \in V^{n}$ such that at least one component of $x^{2}$ is equal to zero.

Now, we give the mentioned characterization.

Theorem 3.2. Let $V$ be a commutative group uniquely divisible by $2, W$ a linear space over the rationals, $n \in \mathbb{N}$ and $k \in\{0, \ldots, n\}$. Then a function $f: V^{n} \rightarrow W$ is $k$-Jensen and $n-k$-quadratic if and only if $f$ satisfies equation (3.1).

Proof. Since for $k \in\{0, n\}$ our assertion follows from [4, Lemma 2.1] (see also [27, Lemma 1.1]) and [31, Theorem 3], we can assume that $k \in\{1, \ldots, n-1\}$.

Let us first suppose that $f: V^{n} \rightarrow W$ is a $k$-Jensen and $n-k$-quadratic mapping. Since then for any $x^{2} \in V^{n-k}$ the mapping $g_{x^{2}}: V^{k} \rightarrow W$ given by

$$
g_{x^{2}}\left(x^{1}\right):=f\left(x^{1}, x^{2}\right), \quad x^{1} \in V^{k},
$$

is $k$-Jensen, [4, Lemma 2.1] (see also [27, Lemma 1.1]) shows that

$$
2^{k} g_{x^{2}}\left(\frac{x_{1}^{1}+x_{2}^{1}}{2}\right)=\sum_{i_{1}, \ldots, i_{k} \in\{1,2\}} g_{x^{2}}\left(x_{1 i_{1}}, \ldots, x_{k i_{k}}\right), \quad x_{1}^{1}, x_{2}^{1} \in V^{k},
$$

which means that

$$
f\left(\frac{x_{1}^{1}+x_{2}^{1}}{2}, x^{2}\right)=2^{-k} \sum_{i_{1}, \ldots, i_{k} \in\{1,2\}} f\left(x_{1 i_{1}}, \ldots, x_{k i_{k}}, x^{2}\right)
$$

for $x_{1}^{1}, x_{2}^{1} \in V^{k}$ and $x^{2} \in V^{n-k}$.

On the other hand, for any $x^{1} \in V^{k}$ the function $h_{x^{1}}: V^{n-k} \rightarrow W$ given by

$$
h_{x^{1}}\left(x^{2}\right):=f\left(x^{1}, x^{2}\right), \quad x^{2} \in V^{n-k},
$$

is $n-k$-quadratic, and therefore from [31, Theorem 3] (see also [18]) it follows that

$$
\sum_{q \in\{-1,1\}^{n-k}} h_{x^{1}}\left(x_{1}^{2}+q x_{2}^{2}\right)=2^{n-k} \sum_{i_{k+1}, \ldots, i_{n} \in\{1,2\}} h_{x^{1}}\left(x_{k+1 i_{k+1}}, \ldots, x_{n i_{n}}\right),
$$

for $x_{1}^{2}, x_{2}^{2} \in V^{n-k}$, which is equivalent to

$$
\sum_{q \in\{-1,1\}^{n-k}} f\left(x^{1}, x_{1}^{2}+q x_{2}^{2}\right)=2^{n-k} \sum_{i_{k+1}, \ldots, i_{n} \in\{1,2\}} f\left(x^{1}, x_{k+1 i_{k+1}}, \ldots, x_{n i_{n}}\right)
$$


for $x_{1}^{2}, x_{2}^{2} \in V^{n-k}$ and $x^{1} \in V^{k}$. Thus, for any $x_{i}^{1}=\left(x_{1 i}, \ldots, x_{k i}\right) \in V^{k}$, $x_{i}^{2}=\left(x_{k+1 i}, \ldots, x_{n i}\right) \in V^{n-k}, i \in\{1,2\}$, equations (3.2) and (3.3) give

$$
\begin{aligned}
& \sum_{q \in\{-1,1\}^{n-k}} f\left(\frac{x_{1}^{1}+x_{2}^{1}}{2}, x_{1}^{2}+q x_{2}^{2}\right) \\
= & 2^{n-k} \sum_{i_{k+1}, \ldots, i_{n} \in\{1,2\}} f\left(\frac{x_{1}^{1}+x_{2}^{1}}{2}, x_{k+1 i_{k+1}}, \ldots, x_{n i_{n}}\right) \\
= & 2^{n-k} \sum_{i_{k+1}, \ldots, i_{n} \in\{1,2\}} 2^{-k} \sum_{i_{1}, \ldots, i_{k} \in\{1,2\}} f\left(x_{1 i_{1}}, \ldots, x_{k i_{k}}, x_{k+1 i_{k+1}}, \ldots, x_{n i_{n}}\right) \\
= & 2^{n-2 k} \sum_{i_{1}, \ldots, i_{n} \in\{1,2\}} f\left(x_{1 i_{1}}, \ldots, x_{n i_{n}}\right),
\end{aligned}
$$

which proves that $f$ satisfies equation (3.1).

Now, assume that $(3.1)$ holds. Putting in it $x_{2}^{2}=(0, \ldots, 0)$ and using Lemma 3.1 we get

$$
2^{k} f\left(\frac{x_{1}^{1}+x_{2}^{1}}{2}, x_{1}^{2}\right)=\sum_{i_{1}, \ldots, i_{k} \in\{1,2\}} f\left(x_{1 i_{1}}, \ldots, x_{k i_{k}}, x_{1}^{2}\right)
$$

for $x_{1}^{1}, x_{2}^{1} \in V^{k}$ and $x_{1}^{2} \in V^{n-k}$, which in view of [4, Lemma 2.1] shows that $f$ is a Jensen mapping in each of the first $k$ variables. Moreover, (3.1) with $x_{1}^{1}=x_{2}^{1}=x^{1}$ gives

$$
\sum_{q \in\{-1,1\}^{n-k}} f\left(x^{1}, x_{1}^{2}+q x_{2}^{2}\right)=2^{n-k} \sum_{i_{k+1}, \ldots, i_{n} \in\{1,2\}} f\left(x^{1}, x_{k+1 i_{k+1}}, \ldots, x_{n i_{n}}\right)
$$

for $x^{1} \in V^{k}, x_{1}^{2}, x_{2}^{2} \in V^{n-k}$, and [31, Theorem 3] now finishes the proof.

\subsection{Stability of equation (3.1) in Banach spaces}

In this subsection, we prove the generalized Hyers-Ulam stability of equation (3.1) in Banach spaces. Our proof is based on Theorem 2.1.

Given a commutative group $V$ which is uniquely divisible by 2 , a linear space $W$ and a function $f: V^{n} \rightarrow W$, we write

$$
\begin{aligned}
(\Phi f)\left(x_{1}^{1}, x_{1}^{2}, x_{2}^{1}, x_{2}^{2}\right):= & \sum_{q \in\{-1,1\}^{n-k}} f\left(\frac{x_{1}^{1}+x_{2}^{1}}{2}, x_{1}^{2}+q x_{2}^{2}\right) \\
& -2^{n-2 k} \sum_{i_{1}, \ldots, i_{n} \in\{1,2\}} f\left(x_{1 i_{1}}, \ldots, x_{n i_{n}}\right)
\end{aligned}
$$

for $x_{1}^{1}, x_{2}^{1} \in V^{k}, x_{1}^{2}, x_{2}^{2} \in V^{n-k}$. Assume also that $k<n$ and let $S$ stand for $\{0,1\}^{n-k}$.

With this notation, we have the following result.

Theorem 3.3. Let $V$ be a commutative group uniquely divisible by $2, W$ a Banach space, $f: V^{n} \rightarrow W, \theta: V^{n} \times V^{n} \rightarrow \mathbb{R}_{+}$. Assume also that for any 


$$
\begin{gathered}
x_{1}^{1}, x_{2}^{1} \in V^{k}, x_{1}^{2}, x_{2}^{2} \in V^{n-k}, \\
\left\|(\Phi f)\left(x_{1}^{1}, x_{1}^{2}, x_{2}^{1}, x_{2}^{2}\right)\right\| \leq \theta\left(x_{1}^{1}, x_{1}^{2}, x_{2}^{1}, x_{2}^{2}\right), \\
\lim _{l \rightarrow \infty}\left(\frac{1}{4^{n-k}}\right)^{l} \sum_{i=0}^{n-k} \sum_{p \in S_{i}}\left(2^{l}-1\right)^{i} \theta\left(x_{1}^{1}, 2^{l} p x_{1}^{2}, x_{2}^{1}, 2^{l} p x_{2}^{2}\right)=0
\end{gathered}
$$

and $\varepsilon^{*}(x)<\infty$ for $x=\left(x^{1}, x^{2}\right) \in V^{n}$, where

$$
\varepsilon^{*}(x):=\sum_{l=0}^{\infty}\left(\frac{1}{4^{n-k}}\right)^{l+1} \sum_{i=0}^{n-k} \sum_{p \in S_{i}}\left(2^{l}-1\right)^{i} \theta\left(x^{1}, 2^{l} p x^{2}, x^{1}, 2^{l} p x^{2}\right) .
$$

Then there exists a unique solution $F: V^{n} \rightarrow W$ of equation (3.1) with

$$
\|f(x)-F(x)\| \leq \varepsilon^{*}(x), \quad x \in V^{n} .
$$

Proof. Putting $x_{1}^{1}=x_{2}^{1}=x^{1} \in V^{k}$ and $x_{1}^{2}=x_{2}^{2}=x^{2} \in V^{n-k}$ in (3.4) we have

$$
\left\|\sum_{s \in S} f\left(x^{1}, 2 s x^{2}\right)-2^{n-2 k} 2^{n} f(x)\right\| \leq \theta(x, x), \quad x=\left(x^{1}, x^{2}\right) \in V^{n},
$$

whence

$$
\left\|\frac{1}{4^{n-k}} \sum_{s \in S} f\left(x^{1}, 2 s x^{2}\right)-f(x)\right\| \leq \frac{1}{4^{n-k}} \theta(x, x), \quad x \in V^{n} .
$$

Define

$$
\mathcal{T} \xi(x):=\frac{1}{4^{n-k}} \sum_{s \in S} \xi\left(x^{1}, 2 s x^{2}\right), \quad \xi \in W^{V^{n}}, x \in V^{n},
$$

and

$$
\varepsilon(x):=\frac{1}{4^{n-k}} \theta(x, x), \quad x \in V^{n} .
$$

Then, by (3.9), we obtain

$$
\|\mathcal{T} f(x)-f(x)\| \leq \varepsilon(x), \quad x \in V^{n} .
$$

Next, put

$$
\Lambda \eta(x):=\frac{1}{4^{n-k}} \sum_{s \in S} \eta\left(x^{1}, 2 s x^{2}\right), \quad \eta \in \mathbb{R}_{+}^{V^{n}}, x \in V^{n} .
$$

It is easily seen that $\Lambda$ has the form described in (H3). Moreover, for any $\xi, \mu \in W^{V^{n}}$ and $x \in V^{n}$ we get

$$
\|\mathcal{T} \xi(x)-\mathcal{T} \mu(x)\| \leq \frac{1}{4^{n-k}} \sum_{s \in S}\left\|\xi\left(x^{1}, 2 s x^{2}\right)-\mu\left(x^{1}, 2 s x^{2}\right)\right\|,
$$

so hypothesis (H2) is also valid. 
Now, using induction, we show that for any $l \in \mathbb{N}_{0}$ and $x=\left(x^{1}, x^{2}\right) \in$ $V^{n}$ we have

$$
\Lambda^{l} \varepsilon(x)=\left(\frac{1}{4^{n-k}}\right)^{l} \sum_{i=0}^{n-k}\left(2^{l}-1\right)^{i} \sum_{p \in S_{i}} \varepsilon\left(x^{1}, 2^{l} p x^{2}\right) .
$$

Fix an $x=\left(x^{1}, x^{2}\right) \in V^{n}$. Since we adopt the convention that $0^{0}=1,(3.12)$ is obvious for $l=0$. Next, assume that (3.12) holds for an $l \in \mathbb{N}_{0}$. Then, applying Lemma 2.2 for $m:=n-k$ and

$$
\varphi(s):=\varepsilon\left(x^{1}, 2^{l+1} s x^{2}\right), \quad s \in S,
$$

we obtain

$$
\begin{aligned}
\Lambda^{l+1} \varepsilon(x) & =\Lambda\left(\Lambda^{l} \varepsilon\right)(x) \\
& =\frac{1}{4^{n-k}} \sum_{v=0}^{n-k} \sum_{u \in S_{v}}\left(\Lambda^{l} \varepsilon\right)\left(x^{1}, 2 u x^{2}\right) \\
& =\left(\frac{1}{4^{n-k}}\right)^{l+1} \sum_{v=0}^{n-k} \sum_{u \in S_{v}} \sum_{w=0}^{n-k}\left(2^{l}-1\right)^{w} \sum_{t \in S_{w}} \varepsilon\left(x^{1}, 2^{l+1} t u x^{2}\right) \\
& =\left(\frac{1}{4^{n-k}}\right)^{l+1} \sum_{v=0}^{n-k} \sum_{w=0}^{n-k} \sum_{t \in S_{w}} \sum_{u \in S_{v}}\left(2^{l}-1\right)^{w} \varepsilon\left(x^{1}, 2^{l+1} t u x^{2}\right) \\
& =\left(\frac{1}{4^{n-k}}\right)^{l+1} \sum_{i=0}^{n-k} \sum_{p \in S_{i}}\left(2^{l+1}-1\right)^{i} \varepsilon\left(x^{1}, 2^{l+1} p x^{2}\right),
\end{aligned}
$$

and thus (3.12) holds for any $l \in \mathbb{N}_{0}$ and $x \in V^{n}$.

Equality (3.12), together with (3.6), shows that all assumptions of Theorem 2.1 are satisfied. Therefore, there exists a unique function $F: V^{n} \rightarrow W$ such that

$$
F(x)=\frac{1}{4^{n-k}} \sum_{s \in S} F\left(x^{1}, 2 s x^{2}\right), \quad x \in V^{n},
$$

and (3.7) holds. Moreover,

$$
F(x)=\lim _{l \rightarrow \infty} \mathcal{T}^{l} f(x), \quad x \in V^{n} .
$$

Now, we show that

$$
\begin{aligned}
& \left\|\Phi\left(\mathcal{T}^{l} f\right)\left(x_{1}^{1}, x_{1}^{2}, x_{2}^{1}, x_{2}^{2}\right)\right\| \\
& \quad \leq\left(\frac{1}{4^{n-k}}\right)^{l} \sum_{i=0}^{n-k} \sum_{p \in S_{i}}\left(2^{l}-1\right)^{i} \theta\left(x_{1}^{1}, 2^{l} p x_{1}^{2}, x_{2}^{1}, 2^{l} p x_{2}^{2}\right)
\end{aligned}
$$

for $l \in \mathbb{N}_{0}, x_{1}^{1}, x_{2}^{1} \in V^{k}$ and $x_{1}^{2}, x_{2}^{2} \in V^{n-k}$. In order to do this, fix $x_{1}^{1}, x_{2}^{1} \in V^{k}$, $x_{1}^{2}, x_{2}^{2} \in V^{n-k}$. If $l=0$, then (3.15) is just (3.4). Next, assume that (3.15) 
holds for an $l \in \mathbb{N}_{0}$. Then

$$
\begin{aligned}
\| \Phi & \left(\mathcal{T}^{l+1} f\right)\left(x_{1}^{1}, x_{1}^{2}, x_{2}^{1}, x_{2}^{2}\right) \| \\
= & \| \sum_{q \in\{-1,1\}^{n-k}} \mathcal{T}^{l+1} f\left(\frac{x_{1}^{1}+x_{2}^{1}}{2}, x_{1}^{2}+q x_{2}^{2}\right) \\
& -2^{n-2 k} \sum_{i_{1}, \ldots, i_{n} \in\{1,2\}} \mathcal{T}^{l+1} f\left(x_{\left.1 i_{1}, \ldots, x_{n i_{n}}\right) \|} \|\right. \\
= & \| \sum_{q \in\{-1,1\}^{n-k}} \frac{1}{4^{n-k}} \sum_{t \in S} \mathcal{T}^{l} f\left(\frac{x_{1}^{1}+x_{2}^{1}}{2}, 2 t x_{1}^{2}+q 2 t x_{2}^{2}\right) \\
& -\frac{2^{n-2 k}}{4^{n-k}} \sum_{i_{1}, \ldots, i_{n} \in\{1,2\}} \sum_{t \in S} \mathcal{T}^{l} f\left(x_{1 i_{1}}, \ldots, x_{k i_{k}}, t\left(2 x_{k+1 i_{k+1}}, \ldots, 2 x_{n i_{n}}\right)\right) \\
= & \left\|\frac{1}{4^{n-k}} \sum_{t \in S} \Phi\left(\mathcal{T}^{l} f\right)\left(x_{1}^{1}, 2 t x_{1}^{2}, x_{2}^{1}, 2 t x_{2}^{2}\right)\right\| \\
\leq & \left(\frac{1}{4^{n-k}}\right)^{l+1} \sum_{t \in S} \sum_{i=0}^{n-k} \sum_{u \in S_{i}}\left(2^{l}-1\right)^{i} \theta\left(x_{1}^{1}, 2^{l+1} t u x_{1}^{2}, x_{2}^{1}, 2^{l+1} t u x_{2}^{2}\right) \\
= & \left(\frac{1}{4^{n-k}}\right)^{l+1} \sum_{i=0}^{n-k} \sum_{p \in S_{i}}\left(2^{l+1}-1\right)^{i} \theta\left(x_{1}^{1}, 2^{l+1} p x_{1}^{2}, x_{2}^{1}, 2^{l+1} p x_{2}^{2}\right) .
\end{aligned}
$$

The last equality follows from Lemma 2.2 with $m:=n-k$ and

$$
\varphi(s):=\theta\left(x_{1}^{1}, 2^{l+1} s x_{1}^{2}, x_{2}^{1}, 2^{l+1} s x_{2}^{2}\right), \quad s \in S .
$$

Letting $l \rightarrow \infty$ in (3.15) and using (3.5) we obtain

$$
(\Phi F)\left(x_{1}^{1}, x_{1}^{2}, x_{2}^{1}, x_{2}^{2}\right)=0,
$$

which means that the function $F$ satisfies equation (3.1).

Finally, assume that $F^{\prime}: V^{n} \rightarrow W$ is another function satisfying equation (3.1) and inequality (3.7), and fix $x \in V^{n}, m \in \mathbb{N}$. Then, by Theorem 3.2, Lemma 2.2 and (3.6), we have

$$
\begin{gathered}
\left\|F(x)-F^{\prime}(x)\right\| \\
=\left\|\left(\frac{1}{4^{n-k}}\right)^{m} F\left(x^{1}, 2^{m} x^{2}\right)-\left(\frac{1}{4^{n-k}}\right)^{m} F^{\prime}\left(x^{1}, 2^{m} x^{2}\right)\right\| \\
\leq\left(\frac{1}{4^{n-k}}\right)^{m}\left(\left\|F\left(x^{1}, 2^{m} x^{2}\right)-f\left(x^{1}, 2^{m} x^{2}\right)\right\|\right. \\
\left.+\left\|F^{\prime}\left(x^{1}, 2^{m} x^{2}\right)-f\left(x^{1}, 2^{m} x^{2}\right)\right\|\right)
\end{gathered}
$$




$$
\begin{aligned}
& \leq\left(\frac{1}{4^{n-k}}\right)^{m} 2 \varepsilon^{*}\left(x^{1}, 2^{m} x^{2}\right) \\
& =2 \sum_{l=0}^{\infty}\left(\frac{1}{4^{n-k}}\right)^{m+l+1} \sum_{i=0}^{n-k} \sum_{p \in S_{i}}\left(2^{l}-1\right)^{i} \theta\left(x^{1}, 2^{m+l} p x^{2}, x^{1}, 2^{m+l} p x^{2}\right) \\
& \leq 2 \sum_{l=m}^{\infty}\left(\frac{1}{4^{n-k}}\right)^{l+1} \sum_{i=0}^{n-k} \sum_{p \in S_{i}}\left(2^{l}-1\right)^{i} \theta\left(x^{1}, 2^{l} p x^{2}, x^{1}, 2^{l} p x^{2}\right) .
\end{aligned}
$$

Consequently, letting $m \rightarrow \infty$ and using the fact that series (3.6) is convergent, we obtain $F(x)=F^{\prime}(x)$, which finishes the proof.

\subsection{Stability of equation (3.1) in complete non-Archimedean normed spaces}

In this subsection, we show the generalized Hyers-Ulam stability of equation (3.1) in complete non-Archimedean normed spaces. The presented result is an analogue of Theorem 3.3, and its proof is based on Theorem 2.3.

Theorem 3.4. Let $V$ be a commutative group uniquely divisible by $2, W$ a complete non-Archimedean normed space over a non-Archimedean field of a characteristic different from $2, f: V^{n} \rightarrow W, \theta: V^{n} \times V^{n} \rightarrow \mathbb{R}_{+}$. Assume also that for any $x_{1}^{1}, x_{2}^{1} \in V^{k}, x_{1}^{2}, x_{2}^{2} \in V^{n-k}$ inequality (3.4) holds and

$$
\lim _{l \rightarrow \infty}\left(\frac{1}{|4|^{n-k}}\right)^{l} \max _{s \in S} \theta\left(x_{1}^{1}, 2^{l} s x_{1}^{2}, x_{2}^{1}, 2^{l} s x_{2}^{2}\right)=0 .
$$

Then there exists a solution $F: V^{n} \rightarrow W$ of equation (3.1) with

$$
\|f(x)-F(x)\| \leq \sup _{l \in \mathbb{N}_{0}}\left(\frac{1}{|4|^{n-k}}\right)^{l+1} \max _{s \in S} \theta\left(x^{1}, 2^{l} s x^{2}, x^{1}, 2^{l} s x^{2}\right), \quad x \in V^{n} .
$$

Proof. Putting $x_{1}^{1}=x_{2}^{1}=x^{1} \in V^{k}$ and $x_{1}^{2}=x_{2}^{2}=x^{2} \in V^{n-k}$ in (3.4) we get (3.8), whence

$$
\left\|\frac{1}{4^{n-k}} \sum_{s \in S} f\left(x^{1}, 2 s x^{2}\right)-f(x)\right\| \leq \frac{1}{|4|^{n-k}} \theta(x, x), \quad x \in V^{n} .
$$

Define an operator $\mathcal{T}$ by (3.10) and put

$$
\varepsilon(x):=\frac{1}{|4|^{n-k}} \theta(x, x), \quad x \in V^{n} .
$$

Then, by (3.18), we obtain (3.11). Next, put

$$
\Lambda \eta(x):=\max _{s \in S} \frac{1}{|4|^{n-k}} \eta\left(x^{1}, 2 s x^{2}\right), \quad \eta \in \mathbb{R}_{+}^{V^{n}}, x \in V^{n} .
$$

It is easily seen that $\Lambda$ has the form described in (N3). Moreover, for any $\xi, \mu \in W^{V^{n}}$ and $x \in V^{n}$ we get

$$
\|\mathcal{T} \xi(x)-\mathcal{T} \mu(x)\| \leq \max _{s \in S} \frac{1}{|4|^{n-k}}\left\|\xi\left(x^{1}, 2 s x^{2}\right)-\mu\left(x^{1}, 2 s x^{2}\right)\right\|,
$$

so hypothesis (N2) is also valid. 
Finally, using induction, one can check that for any $l \in \mathbb{N}$ and $x \in V^{n}$, we have

$$
\Lambda^{l} \varepsilon(x)=\max _{s \in S}\left(\frac{1}{|4|^{n-k}}\right)^{l} \varepsilon\left(x^{1}, 2^{l} s x^{2}\right),
$$

which, together with (3.16), shows that all assumptions of Theorem 2.3 are satisfied. Therefore, there exists a function $F: V^{n} \rightarrow W$ such that (3.13) and (3.17) hold. Moreover, the function $F$ is given by formula (3.14).

Now, we show that

$$
\begin{aligned}
\| \Phi\left(\mathcal{T}^{l} f\right) & \left(x_{1}^{1}, x_{1}^{2}, x_{2}^{1}, x_{2}^{2}\right) \| \\
& \leq\left(\frac{1}{|4|^{n-k}}\right)^{l} \max _{s \in S} \theta\left(x_{1}^{1}, 2^{l} s x_{1}^{2}, x_{2}^{1}, 2^{l} s x_{2}^{2}\right)
\end{aligned}
$$

for $l \in \mathbb{N}_{0}, x_{1}^{1}, x_{2}^{1} \in V^{k}$ and $x_{1}^{2}, x_{2}^{2} \in V^{n-k}$. In order to do this, fix $x_{1}^{1}, x_{2}^{1} \in V^{k}$, $x_{1}^{2}, x_{2}^{2} \in V^{n-k}$. If $l=0$, then (3.20) follows immediately from (3.4). Next, assume that (3.20) holds for an $l \in \mathbb{N}_{0}$. Then

$$
\begin{aligned}
\| \Phi\left(\mathcal{T}^{l+1} f\right) & \left(x_{1}^{1}, x_{1}^{2}, x_{2}^{1}, x_{2}^{2}\right) \| \\
= & \left\|\frac{1}{4^{n-k}} \sum_{t \in S} \Phi\left(\mathcal{T}^{l} f\right)\left(x_{1}^{1}, 2 t x_{1}^{2}, x_{2}^{1}, 2 t x_{2}^{2}\right)\right\| \\
& \leq\left(\frac{1}{|4|^{n-k}}\right)^{l+1} \max _{s \in S} \theta\left(x_{1}^{1}, 2^{l+1} s x_{1}^{2}, x_{2}^{1}, 2^{l+1} s x_{2}^{2}\right) .
\end{aligned}
$$

Letting $l \rightarrow \infty$ in (3.20) and using (3.16) we obtain that the function $F$ satisfies equation (3.1).

\section{Applications}

A consequence of Theorem 3.3 is the following result on the classical HyersUlam stability of equation (3.1).

Corollary 4.1. Assume that $\varepsilon>0, n>k, V$ is a commutative group uniquely divisible by 2 and $W$ is a Banach space. If $f: V^{n} \rightarrow W$ satisfies, for any $x_{i}^{1} \in V^{k}, x_{i}^{2} \in V^{n-k}, i \in\{1,2\}$, the inequality

$$
\left\|(\Phi f)\left(x_{1}^{1}, x_{1}^{2}, x_{2}^{1}, x_{2}^{2}\right)\right\| \leq \varepsilon
$$

then there exists a unique solution $F: V^{n} \rightarrow W$ of equation (3.1) such that

$$
\|f(x)-F(x)\| \leq \frac{\varepsilon}{2^{n-k}\left(2^{n-k}-1\right)}, \quad x \in V^{n} .
$$

Proof. In order to apply Theorem 3.3 with $\theta \equiv \varepsilon$, it suffices to show that condition (3.5) holds and $\varepsilon^{*}(x)<\infty$ for $x \in V^{n}$, where $\varepsilon^{*}(x)$ is given by (3.6).

To this end, let us first note that for any $l \in \mathbb{N}$ and $x_{1}^{1}, x_{2}^{1} \in V^{k}$, $x_{1}^{2}, x_{2}^{2} \in V^{n-k}$ we have

$$
\sum_{i=0}^{n-k} \sum_{p \in S_{i}}\left(2^{l}-1\right)^{i} \theta\left(x_{1}^{1}, 2^{l} p x_{1}^{2}, x_{2}^{1}, 2^{l} p x_{2}^{2}\right)=\varepsilon 2^{l(n-k)} .
$$


Since

$$
\lim _{l \rightarrow \infty}\left(\frac{1}{4^{n-k}}\right)^{l} \varepsilon 2^{l(n-k)}=\varepsilon \lim _{l \rightarrow \infty}\left(\frac{1}{2^{n-k}}\right)^{l}=0,
$$

condition (3.5) is fulfilled. Moreover, for any $x \in V^{n}$,

$$
\begin{aligned}
\varepsilon^{*}(x) & =\sum_{l=0}^{\infty}\left(\frac{1}{4^{n-k}}\right)^{l+1} \varepsilon 2^{l(n-k)} \\
& =\frac{\varepsilon}{4^{n-k}} \sum_{l=0}^{\infty}\left(\frac{1}{2^{n-k}}\right)^{l} \\
& =\frac{\varepsilon}{2^{n-k}\left(2^{n-k}-1\right)}<\infty
\end{aligned}
$$

which completes the proof.

Similarly, we may apply Theorem 3.4 to the case of the control function

$$
\theta\left(x_{1}^{1}, x_{1}^{2}, x_{2}^{1}, x_{2}^{2}\right)=\prod_{j=1}^{n}\left\|x_{j 1}\right\|^{r_{j 1}} \cdot\left\|x_{j 2}\right\|^{r_{j 2}}
$$

with some additional assumptions. Consequently, we have the following result.

Corollary 4.2. Assume that $n>k, r_{j 1}, r_{j 2} \in \mathbb{R}$ for $j \in\{1, \ldots, n\}$ are positive real numbers with $\sum_{j=k+1}^{n}\left(r_{j 1}+r_{j 2}\right)>2 n-2 k, V$ is a normed space and $W$ is a complete non-Archimedean normed space over a non-Archimedean field of a characteristic different from 2 such that $|2|<1$. If $f: V^{n} \rightarrow W$ satisfies, for any $x_{i}^{1} \in V^{k}, x_{i}^{2} \in V^{n-k}, i \in\{1,2\}$, the inequality

$$
\left\|(\Phi f)\left(x_{1}^{1}, x_{1}^{2}, x_{2}^{1}, x_{2}^{2}\right)\right\| \leq \prod_{j=1}^{n}\left\|x_{j 1}\right\|^{r_{j 1}} \cdot\left\|x_{j 2}\right\|^{r_{j 2}},
$$

then there exists a solution $F: V^{n} \rightarrow W$ of equation (3.1) with

$$
\|f(x)-F(x)\| \leq \frac{1}{|4|^{n-k}} \prod_{j=1}^{n}\left\|x_{j}\right\|^{r_{j 1}+r_{j 2}}, \quad x=\left(x_{1}, \ldots, x_{n}\right) \in V^{n} .
$$

\section{Conclusion}

In this paper, we reduce the system of $n$ equations defining the multi-Jensenquadratic mapping to a single functional equation and we prove, using the fixed point method, the generalized (in the spirit of D. G. Bourgin and P. Găvruţa) Hyers-Ulam stability of this equation both in Banach spaces and in complete non-Archimedean normed spaces. Our results are significant supplements and/or generalizations of some results from $[1,12,13,14,16$, $18,23,27,29,30,31]$. 


\section{References}

[1] J.-H. Bae and W.-G. Park, On a cubic equation and a Jensen-quadratic equation. Abstr. Appl. Anal. 2007 (2007), Article ID 45179.

[2] A. Bahyrycz, J. Brzdęk, E. Jabłońska and J. Olko, On functions that are approximate fixed points almost everywhere and Ulam's type stability. J. Fixed Point Theory Appl. 17 (2015), 659-668.

[3] A. Bahyrycz, K. Ciepliński and J. Olko, On an equation characterizing multiadditive-quadratic mappings and its Hyers-Ulam stability. Appl. Math. Comput. 265 (2015), 448-455.

[4] A. Bahyrycz, K. Ciepliński and J. Olko, On an equation characterizing multiCauchy-Jensen mappings and its Hyers-Ulam stability. Acta Math. Sci. Ser. B Engl. Ed. 35 (2015), 1349-1358.

[5] N. Brillouët-Belluot, J. Brzdęk and K. Ciepliński, On some recent developments in Ulam's type stability. Abstr. Appl. Anal. 2012 (2012), Article ID 716936.

[6] J. Brzdęk, L. Cădariu and K. Ciepliński, Fixed point theory and the Ulam stability. J. Funct. Spaces 2014 (2014), Article ID 829419.

[7] J. Brzdęk, J. Chudziak and Zs. Páles, A fixed point approach to stability of functional equations. Nonlinear Anal. 74 (2011), 6728-6732.

[8] J. Brzdęk and K. Ciepliński, A fixed point approach to the stability of functional equations in non-Archimedean metric spaces. Nonlinear Anal. 74 (2011), 68616867.

[9] J. Brzdęk and K. Ciepliński, Remarks on the Hyers-Ulam stability of some systems of functional equations. Appl. Math. Comput. 219 (2012), 4096-4105.

[10] J. Brzdęk and K. Ciepliński, Hyperstability and superstability. Abstr. Appl. Anal. 2013 (2013), Article ID 401756.

[11] J. Brzdęk, K. Ciepliński and Z. Leśniak, On Ulam's type stability of the linear equation and related issues. Discrete Dyn. Nat. Soc. 2014 (2014), Article ID 536791.

[12] K. Ciepliński, On multi-Jensen functions and Jensen difference. Bull. Korean Math. Soc. 45 (2008), 729-737.

[13] K. Ciepliński, Stability of the multi-Jensen equation. J. Math. Anal. Appl. 363 (2010), 249-254.

[14] K. Ciepliński, On the generalized Hyers-Ulam stability of multi-quadratic mappings. Comput. Math. Appl. 62 (2011), 3418-3426.

[15] K. Ciepliński, Applications of fixed point theorems to the Hyers-Ulam stability of functional equations - A survey. Ann. Funct. Anal. 3 (2012), 151-164.

[16] K. Ciepliński and A. Surowczyk, On the Hyers-Ulam stability of an equation characterizing multi-quadratic mappings. Acta Math. Sci. Ser. B Engl. Ed. 35 (2015), 690-702.

[17] A. G. Ghazanfari and Z. Alizadeh, On approximate ternary $m$-derivations and o-homomorphisms. J. Fixed Point Theory Appl. 17 (2015), 625-640.

[18] P. Ji, W. Qi and X. Zhan, Generalized stability of multi-quadratic mappings. J. Math. Res. Appl. 34 (2014), 209-215.

[19] S.-M. Jung, Hyers-Ulam-Rassias Stability of Functional Equations in Nonlinear Analysis. Springer, New York, 2011. 
[20] S.-M. Jung, D. Popa and M. Th. Rassias, On the stability of the linear functional equation in a single variable on complete metric groups. J. Global Optim. 59 (2014), 165-171.

[21] Pl. Kannappan, Functional Equations and Inequalities with Applications. Springer, New York, 2009.

[22] A. Khrennikov, Non-Archimedean Analysis: Quantum Paradoxes, Dynamical Systems and Biological Models. Kluwer Academic Publishers, Dordrecht, 1997.

[23] K.-H. Kim, J.-H. Bae and W.-G. Park, Stability of a system of functional equations on Jensen-quadratic mappings. J. Chungcheong Math. Soc. 22 (2009), $31-37$.

[24] M. Kuczma, An Introduction to the Theory of Functional Equations and Inequalities. Cauchy's Equation and Jensen's Inequality. Birkhäuser Verlag, Basel, 2009.

[25] M. S. Moslehian and Th. M. Rassias, Stability of functional equations in nonArchimedean spaces. Appl. Anal. Discrete Math. 1 (2007), 325-334.

[26] W. Prager and J. Schwaiger, Multi-affine and multi-Jensen functions and their connection with generalized polynomials. Aequationes Math. 69 (2005), 41-57.

[27] W. Prager and J. Schwaiger, Stability of the multi-Jensen equation. Bull. Korean Math. Soc. 45 (2008), 133-142.

[28] P. K. Sahoo and Pl. Kannappan, Introduction to Functional Equations. CRC Press, Boca Raton, FL, 2011.

[29] J. Schwaiger, Some remarks on the stability of the multi-Jensen equation. Cent. Eur. J. Math. 11 (2013), 966-971.

[30] T. Z. Xu, Stability of multi-Jensen mappings in non-Archimedean normed spaces. J. Math. Phys. 53 (2012), 023507.

[31] X. Zhao, X. Yang and C.-T. Pang, Solution and stability of the multiquadratic functional equation. Abstr. Appl. Anal. 2013 (2013), Article ID 415053.

\author{
Anna Bahyrycz \\ Faculty of Applied Mathematics \\ AGH University of Science and Technology \\ Mickiewicza 30 \\ 30-059 Kraków \\ Poland \\ e-mail: bah@up.krakow.pl \\ Krzysztof Ciepliński \\ Faculty of Applied Mathematics \\ AGH University of Science and Technology \\ Mickiewicza 30 \\ 30-059 Kraków \\ Poland \\ e-mail: cieplin@agh.edu.pl
}

Open Access This article is distributed under the terms of the Creative Commons Attribution 4.0 International License (http://creativecommons.org/licenses/by/4.0/), which permits unrestricted use, distribution, and reproduction in any medium, provided you give appropriate credit to the original author(s) and the source, provide a link to the Creative Commons license, and indicate if changes were made. 